Ökologie, Wettbewerbsfähigkeit und Erfolgsbedingungen ökologischer Wettbewerbsstrategien

\section{Strategisch gestaltbares Verhältnis}

\section{Die wirtschaftliche und strategische Relevanz der Ökologie wird zumeist unter- schätzt. Die Ökologie kann sowohl als unerwünschter Nachteil oder Bedrohung der Wettbewerbsfähigkeit als auch als erwünschter Vorteil und Chance erschei- nen. Diese Ambivalenz läßt sich auf operativer wie auch auf strategischer Hand- lungsebene feststellen und führt zu entsprechend unterschiedlichen Strategien.}

$\mathrm{U}$ Von Thomas Dyllick mweltschutz wird oftmals immer noch als Kostenfaktor gesehen, weshalb entsprechende Maßnahmen so weit und so lange wie möglich vermieden werden. Erst sehr zögerlich breitet sich eine andere Perspektive aus, die in Abfällen und Emissionen vor allem fehlgeleitete und verschwendete Ressourcen sieht, was nicht nur ökologisch, sondern auch ökonomisch unsinnig ist. In dieser neuen Perspektive dient Umweltmanagement der gezielten Verbesserung der Ressourceneffizienz. Ökologie und Umweltmanagement sind hier kein unerwünschter Kostenfaktor, sondern ein ökonomisch erwïnschter Produktivitätsfaktor.

Verlagert man den Blick von der operativen auf die strategische Ebene, so erscheint auch hier die ökologie in zweifacher Form. Einerseits ist sie mit vielfältigen Risiken für bestehende Produkte und Technologien verbunden, wenn man z.B. an das Verbot von Stoffen wie FCKW oder die Ächtung von Produkten aus PVC denkt. Andererseits bietet sie aber auch Differenzierungs- und Profilierungsmöglichkeiten, Beispiele sind die Entwicklung von chlorfrei gebleichtem Papier, Möglichkeiten ökologischen Bauens oder Lebensmittel und Textilien aus biologischem Anbau.

\section{Vier Strategietypen}

Neben dem Marktbezug von ökologischen Wettbewerbsstrategien, zu denken ist hier an $\mathrm{Ab}$ satz-, aber auch an Personal- und Finanzmärkte, ist immer auch der Gesellschaftsbezug, d.h. Politik und Öffentlichkeit, zu berücksichtigen. Daneben ist zwischen einer offensiven und einer defensiven Strategieausrichtung zu unterscheiden. Hieraus ergeben sich vier Typen ökologischer Wettbewerbsstrategien (vgl. Abb. 1) (1). Ökologische Marktabsicherungsstrategien entspringen einer defensiven Ausrichtung und reagieren auf gesellschaftliche Einflüsse, die bestehende Märkte und Geschäftstätigkeiten zu gefährden drohen. Ziel dieser Strategie ist es, ökologisch „clean“ zu sein, um sich gegenüber ökologischen Ansprüchen abzusichern, noch bevor diese wettbewerbsrelevant werden. Durch geeignete Maßnahmen, wie bspw. Kommunikation oder Selbstverpflichtungen, wird versucht Ansprïche zu kontrollieren oder ihnen entgegenzukommen. So bekennen sich z.B. die Lebensmittelgroßverteiler dazu, ihre Angebote so lange wie möglich gentechnikfrei $\mathrm{zu}$ halten, um negative Kundenreaktionen zu vermeiden. Die Chemieunternehmen verpflichten sich im Rahmen von „Responsible Care“ zu einem umfassenden Umwelt- und Sicherheitsmanagement, um bestehende Risiken zu minimieren und das Image in der Öffentlichkeit zu verbessern.

Ebenso wie ökologische Marktabsicherungsstrategien sind auch ökologische Kostenstrategien Ausdruck einer defensiven Ausrichtung. In diesem Fall versuchen Untenehmen einmal bestehende ökologische Anforderungen möglichst „effizient“, d.h. kostengünstig zu erfüllen. In der Lebensmittelbranche sind hier Maßnahmen zur Verbesserung der Energie- und Wassereffizienz oder Verpackungsoptimierungen zu nennen. In der Landwirtschaft spielen die ökologischen
Ausgleichszahlungen eine Rolle. Für das Energiemanagement ist das Potential professioneller Lösungen im Rahmen eines Energie-Contracting von besonderer ökologischer und ökonomischer Bedeutung. Ein interessantes Beispiel ist auch die Brennstoffsubstitution durch diverse Abfälle in der schweizerischen Zementindustrie. Erst jüngst hat eine Befragung der Arbeitsgemeinschaft Selbständiger Unternehmer in Deutschland belegt, daß auch der Einsatz eines Umweltmanagementsystems (UMS) kosteneffizient ist. Für knapp die Hälfte der befragten Unternehmen beträgt die Amortisationszeit für den UMS-Aufbau weniger als eineinhalb Jahre. UMS erscheinen demnach rein ökonomisch als interessante Investitionen zur Verbesserung der Ressourceneffizienz (2).

Ökologische Differenzierungsstrategien sind das Resultat einer offensiven Ausrichtung von Unternehmen und beziehen sich primär auf den Markt. Das Ziel besteht darin, durch Ökologie Innovationspotentiale zu erschließen, d.h. auf dem Markt „innovativ“ zu sein. Gerade in gesättigten Märkten, auf denen sich die Produkte zunehmend angleichen und aus der Sicht des Konsumenten austauschbar werden, erweist sich die Ökologie als ein interessantes Differenzierungskriterium. So spielen Bio-Produkte in der Lebensmittel- und Textilbranche eine besondere Rolle als ökologische Differenzierungsstrategie, aber auch regionale Produkte und ÖkoLabels zur Kommunikation ökologischer Mehrleistungen. Im Baubereich ist an superisolierende Fenster und Fassadensysteme zu denken, an Baustoffe aus Recyclingmaterialien oder an komplexe Innovationen wie das ,Minergiehaus“. In neu entstehenden ökologischen Märk-

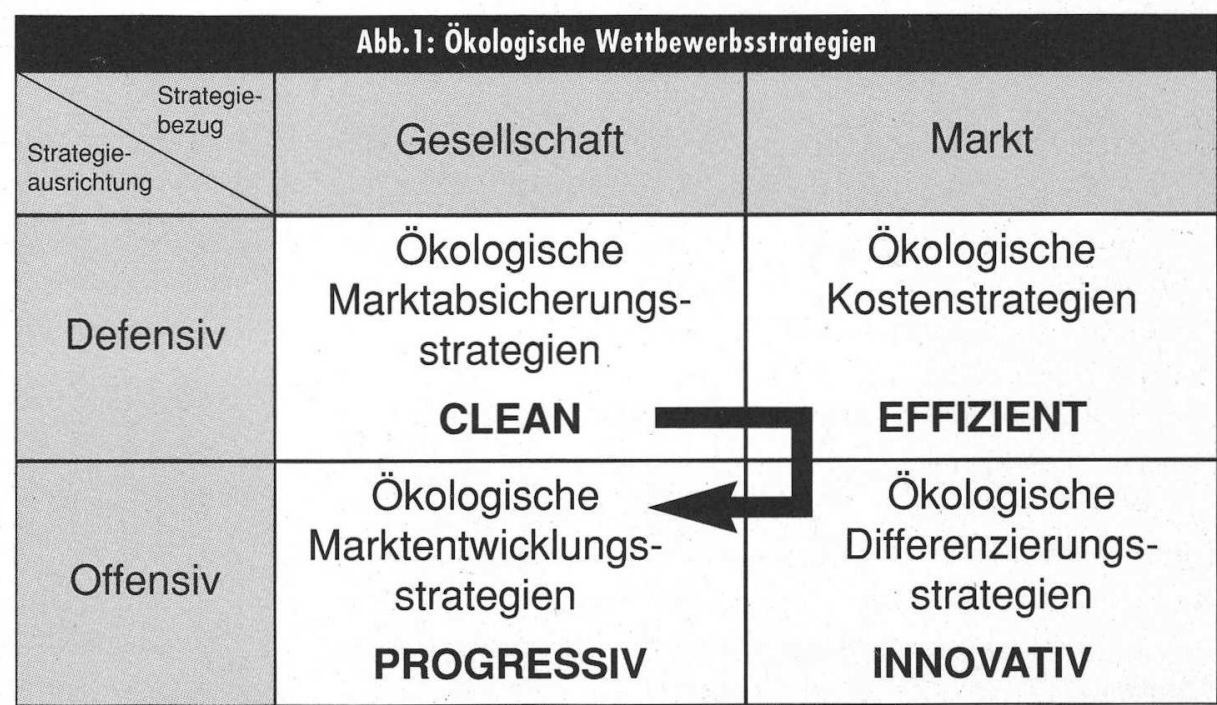

Quelle: Dyllick/Belz/Schneidewind 1997 
ten besteht für Unternehmen die Möglichkeit, sich durch ökologische Produkte oder Dienstleistungen zu positionieren. Dies betrifft z.B. im Falle der Lebensmittel das rasch wachsende Segment vegetarischer Produkte; bezüglich Altlasten entsteht ein neuer Markt für effiziente Sanierungstechnologien.

Eine Gefahr von Öko-Produkten und Öko-Märkten besteht darin, daß sie auf Dauer in Nischen verharren. Ein Beispiel ist der Markt für Sonnenkollektoren. Obwohl ökologisch sinnvoll und technisch machbar, haben sich Sonnenkollektoren bisher nicht auf breiter Front durchsetzen können. Dazu bedarf es einer Änderung der politischen Rahmenbedingungen.

Dies ist der Ansatzpunkt von ökologischen Marktentwicklungsstrategien. Sie entspringen einer offensiven Ausrichtung und beziehen sich auf die Rahmenbedingungen, innerhalb derer Wettbewerb stattfindet. Das Ziel besteht darin, die Voraussetzungen mitzugestalten, die zur Entstehung und Vergrößerung ökologischer Wettbewerbsfelder führen. Strategien dieser Art sollen als "progressiv" bezeichnet werden. Sie zielen darauf ab, Öffentlichkeit, Politik oder Marktumfeld gezielt zu beeinflussen. Ein gutes Beispiel hierfür sind die flankierenden Maßnahmen der schweizerischen Zementindustrie, auf deren Anregung eine Arbeitsgruppe des Bundesamtes für Umwelt, Wald und Landschaft sich an die Arbeit gemacht hat, um Kriterien und Regeln für die Abfallentsorgung in Zementwerken zu erarbeiten. Hiermit wurde die Möglichkeit zur Abfallverbrennung verbindlich geregelt und die Brennstoffsubstitutionsstrategie der Zementin- dustrie politisch legitimiert. Daneben wurden Absprachen mit den aufgeschreckten Betreibern von Hausmüllverbrennungsanlagen getroffen.

\section{Strategiebezogene Lernprozesse}

Festzuhalten ist, daß die Strategien den Charakter von Idealtypen haben. Demgegenüber handelt es sich im Falle von realtypischen Strategien oftmals um komplexe Strategien, die unterschiedliche Elemente miteinander kombinieren. Der vermittels des Pfeils in der Abbildung angedeutete Ablauf bezieht sich auf typische unternehmensspezifische Lemprozesse. Demnach bringen ökologische Marktabsicherungsstrategien die zunächst festzustellende Abwehrhaltung von Unternehmen zum Ausdruck, wenn sie mit ungewohnten ökologischen Forderungen konfrontiert werden. Kostenstrategien setzen typischerweise dann ein, wenn sich Unternehmen in das Schicksal fügen, das ihnen durch ökologische Auflagen oder Anforderungen auferlegt wird. Sie versuchen das ökonomisch Beste aus dieser Situation zu machen. Dabei passiert es oftmals, daß erst im Zuge von extern induzierten Umweltaktivitäten das Kostensenkungspotential überhaupt erkannt wird, womit eine Entwicklung in Richtung offensives Ressourcen- und Kostenmanagement ausgelöst wird. Dies kann z.B. im Zuge des Einsatzes von Umweltmanagementsystemen festgestellt werden. Ökologische Differenzierungsstrategien stellen ein Entwicklungspotential für Unternehmen dar, die ihre Spielräume für Kostensenkungen bereits ausgeschöpft haben. Sie bedingen jedoch eine ökologische Sensibilität und Reife des Absatzmarktes. Zudem ist davon auszugehen, daß die Strategien und Merkmale ökologischer Differenzierungsstrategien ganz anders geartet sind, je nachdem ob sie auf eine Öko-Nische oder den ökologischen Massenmarkt ausgerichtet sind. Nicht nur die Kundensegmente (Öko-Aktive vs. Öko-Normalbürger) und Vertriebswege (Öko-Läden vs. Supermarkt) unterscheiden sich deutlich voneinander, sondern auch die Ansprüche an die ökologische Qualität bzgl. Produkten und Herstellung, die Preissensibilität sowie die Akzeptanz und Wirksamkeit von Werbemaßnahmen (3). Ökologische Marktentwicklungsstrategien werden schließlich nur von wenigen, zumeist ökologisch fortschrittlichen Unternehmen ergriffen, die sich als ,strukturpolitische Akteure" (4) verstehen.

\section{Anmerkungen}

(1) Vgl. zu den hier entwickelten Zusammenhängen sowie der Strategietypologie T. Dyllick/ F. Belz/ U. Schneidewind: Ökologie und Wettbewerbsfähigkeit. Hanser, München und NZZ Buchverlag, Zürich 1997.

(2) Vgl. K. Günther: Betriebliches Umweltmánagement setzt sich in der Praxis durch. In: UmweltWirtschaftsForum, 6. Jg., Heft 1, März 1998, S. 18.

(3) Vgl. A. Villiger/ R. Wüstenhagen/ A. Meyer: Jenseits der Öko-Nische. Haupt, Bern u.a. (im Erscheinen).

(4) Vgl. U. Schneidewind: Die Unternehmung als strukturpolitischer Akteur. Metropolis, Marburg 1998.

\section{Der Autor}

Prof. Dr. Thomas Dyllick ist Direktor des Instituts für Wirtschaft und Ökologie der Universität St. Gallen. Kontakt: IWÖ-HSG, Tigerbergstr. 2, CH-9000 St. Gallen, Tel. $0041 / 71 / 224-2596$, Fax - 2722, E-mail:Thomas.Dyllick@unisg.ch

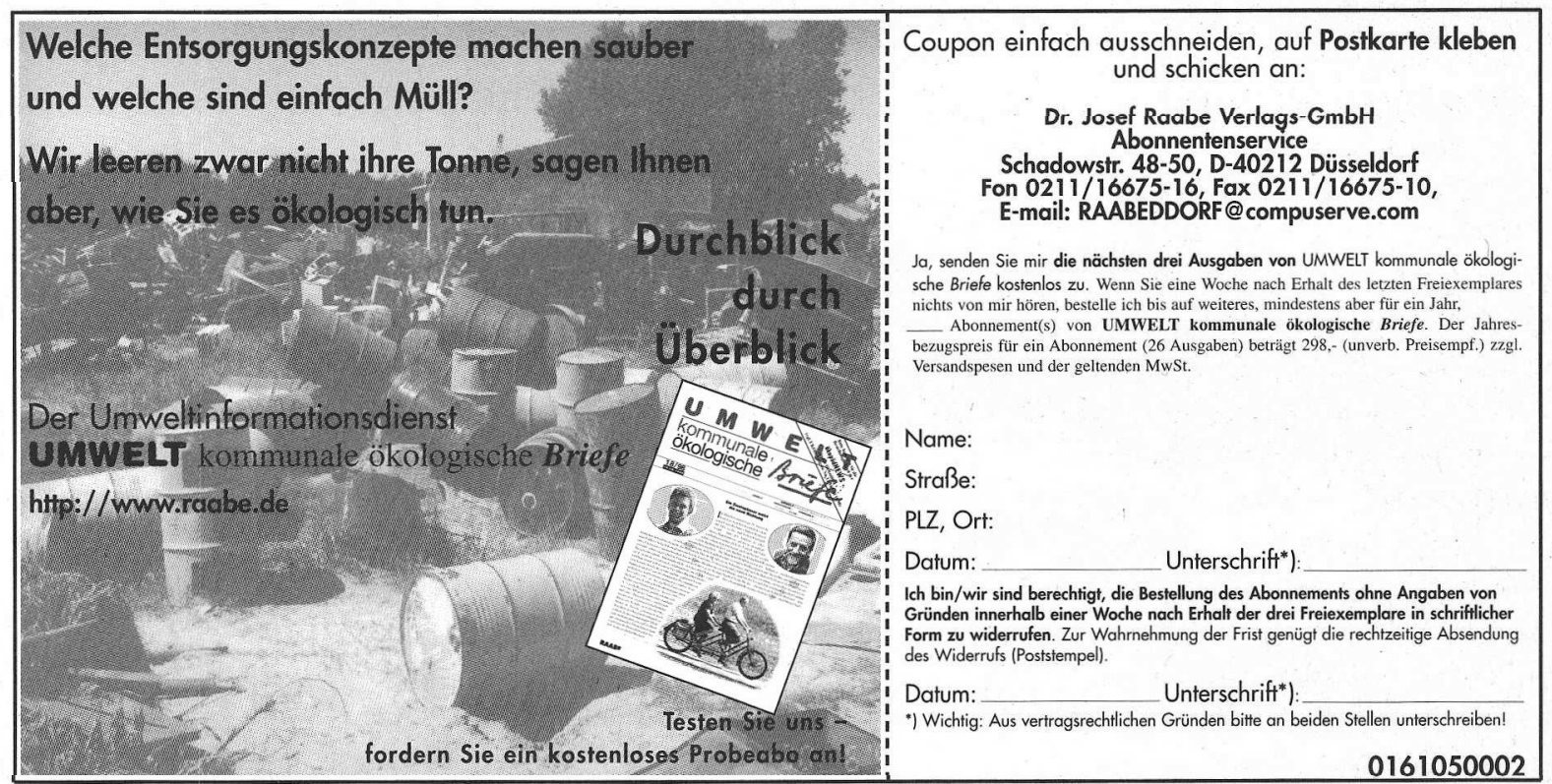


(c) 20I0 Authors; licensee IÖW and oekom verlag. This is an article distributed under the terms of the Creative Commons Attribution Non-Commercial No Derivates License (http://creativecommons.org/licenses/by-nc-nd/3.o/), which permits unrestricted use, distribution, and reproduction in any medium, provided the original work is properly cited. 